\title{
注意力机制在自然场景文字检测中的应用
}

\author{
王延昭，顾晓东* \\ (复旦大学信息科学与工程学院电子工程系 上海 200438) \\ (xdgu@fudan.edu.cn)
}

\begin{abstract}
摘 要: 针对目前主流的基于分割的文字检测方法中由于需要复杂的后处理过程保证检测精度，通常难以实现高检 测速度的问题，提出一种应用位置注意力模块和金字塔注意力网络 2 种注意力机制的方法. 首先用金字塔注意力网 络对图像进行特征提取及语义分割; 同时将位置注意力模块应用于高层特征，通过加强图像中相似物体的权重加强 对文字的检测效果; 最后进行简单有效的后处理, 在实现较高检测准确度的前提下提高检测速度. 实验结果表明, 在 Total-text 数据集中, 采用更轻量化的骨干网络时, 所提方法在检测速度上优势明显; 采用更深层的骨干网络时, 所提方法的检测准确度领先 $2.0 \%$.
\end{abstract}

关键词：自然场景文字检测；神经网络；金字塔注意力网络；位置注意力机制

中图法分类号: TP391.41 DOI: 10.3724/SP.J.1089.2021.18700

\section{Using of Attention for Scene Text Detection}

\author{
Wang Yanzhao and Gu Xiaodong* \\ (Department of Electronic Engineering, School of Information Science and Technology, Fudan University, Shanghai 200438)
}

\begin{abstract}
In view of the issue that current mainstream segmentation-based text detection methods is difficult to achieve high detection speed due to complex post-processing to ensure detection accuracy, a scene text detection method is proposed which applies pyramid attention network and position attention module. First, it adopts pyramid attention network to perform feature extraction and semantic segmentation. Meanwhile, it adopts position attention module in high-level features, which strengthens the weights of similar objects in the image to enhance the effect of text detection. Finally, it adopts a simple and effective post-processing algorithm to increase detection speed under the premise of high detection accuracy. Experimental results show that in Total-text datasets, using light-weight backbone network, the method has great advantages on detection speed, and while using deeper backbone network, the method achieves the state of the art result and has a $2.0 \%$ lead on detection accuracy.
\end{abstract}

Key words: scene text detection; neural network; pyramid attention network; position attention module

近年来，自然文字阅读成为人们关注的热点， 它在很多场景中有着广泛应用, 包括产品搜索、图 像与视频理解、盲人辅助和自动驾驶等. 自然场景 文字检测是这些应用中重要的一个步骤, 它的准
确程度是后续自然场景文字识别效果的保证. 同 时, 自然文字检测也是面临着最大挑战的一个步 骤，这些挑战包括文字大小不一、长宽比例不一、 方向形状各异, 以及各种自然条件的影响. 以往采

收稿日期: 2020-10-08; 修回日期: 2021-09-10. 基金项目：国家自然科学基金面上项目(61771145). 王延昭(1994一), 男, 硕士研 究生, 主要研究方向为基于神经网络的自然场景文字检测与识别; 顾晓东(1970-), 男, 博士, 教授, 博士生导师, 论文通讯作者, 主 要研究方向为人工神经网络、深度学习、智能信号处理、仿生建模、跨模态分析. 
用的检测方法大多是基于回归的方法，其最大的 问题在于无法有效地检测各种特殊形状的文字区 域，尤其是在自然场景中出现频率很高的曲文. 随 着深度学习中对图像分割的研究逐渐加深, 自然 场景文字检测取得了进展和突破，因为基于分割 的方法可以得到像素级的预测结果，所以其适用 于检测任意形状的文字区域. 然而，由于这类方法 需要在后处理过程中将像素级的预测结果整合为 文字区域，而为了确保检测结果的准确度，采用的 后处理算法往往比较复杂, 且在二值化过程中國 值的选择往往对检测结果影响较大, 这就降低了 方法的检测速度.

以较为经典、具有较高检测准确度的渐进式尺 度扩展网络(progressive scale expansion network, PSENet $)^{[1]}$ 方法为例，在制作标签时，采用 Vatti 裁 剪算法 ${ }^{[2]}$ 制作了 7 个具有不同收缩比例的标签(称 之内核). 这些内核有 2 个作用: 一是用不同收缩 比例得到的内核分别作为每幅特征图中的 Ground Truth，通过适当的权重优化检测结果；二是用对 应最小尺寸的内核的检测结果作为原始检测结果, 使用基于宽度优先搜索的渐进的尺度扩展算法来 膨胀得到最终的结果. PSENet 有着较高的检测准 确度，也可以解决很近的 2 个文字区域被错误检测 为一个文字区域的情况，然而，该方法最大的不足 在于复杂的后处理过程大大增加了检测时间.

本文以另一种思路使用 Vatti 裁剪算法, 将原 始的 Ground Truth 以 0.7 为收缩比例裁剪为标签, 并以由该标签得到的对应检测结果为内核，使用 Vatti 裁剪算法进行膨胀. 这一后处理过程耗时很 少，极大地增强了检测的速度. 同时，本文将应用 注意力机制提升检测准确度，以弥补简单后处理 过程带来准确度的下降.

传统场景文字检测方法的局限性在于对小尺 寸文字的检测精度较低，容易漏检. 位置注意力模 块(position attention module, PAM) ${ }^{[3]}$ 是一种通过引 人自注意力机制捕获特征图任意 2 个位置之间的 空间依赖性的注意力模块，旨在利用图像中 2 个 位置的特征之间的关联，使具有相似特征的任意 2 个位置相互增强各自特征的表达. 针对自然场景 文字所占图像比例较小，同一图像中文字区域可 能较多, 以及多个文字区域可能有相同或近似特 征的特点, PAM 可以有效地增强图像中的文字像 素，使文字像素和非文字像素更易被区分，提升检 测的准确度.

金字塔注意力网络(pyramid attention network,
PAN $)^{[4]}$ 是一种以金字塔结构为基础的特征提取网 络，与广泛使用的特征金字塔网络(feature pyramid network, FPN)相比，其引人了注意力机制，具有更 好的特征提取效果. PAN 包含了特征金字塔注意力 (feature pyramid attention, FPA)和全局注意力上采 样(global attention upsample, GAU) 2 种模块; 其 中, FPA 能够提供像素级注意力信息, 并通过金字 塔结构扩大感受野的范围, GAU 能够利用高层次 特征图来指导低层次特征恢复图像像素的定位.

自适应二值化算法 ${ }^{[5]}$ 是一种新颖的将像素预 测结果图转化为二值化检测结果图的算法. 不同 于传统算法使用固定阈值获得二值化图，导致阈 值的不同对性能影响很大，自适应二值化算法对 每一个像素点进行自适应二值化, 二值化阈值由 网络学习得到, 使最终的输出图对于阈值有着很 高的鲁棒性.

本文提出将注意力机制应用于自然场景文字检 测中，包括用 PAM 和 PAN 提升检测的准确度. 同时, 本文还采用了并不复杂的后处理过程, 以保证获得 较高检测准确度的同时达到较快的检测速度.

\section{1 相关工作}

近年来，已有多种多样的自然场景文字检测 方法，它们大致可分为基于回归的方法和基于分 割的方法 2 类.

基于回归的方法采用直接回归文字区域边界 框或四边形文字区域顶点位置的方法得到检测结 果. 单发多框检测器(single shot multibox detector, $\mathrm{SSD})^{[6]}$ 是一个以回归文字区域边界框为思路实现 的优秀方法. TextBoxes ${ }^{++}{ }^{[7]}$ 以 SSD 为基础, 改进了 针框的长宽比设置和卷积核的尺寸, 并采用四边 形边界框回归代替传统的矩形边界框回归, 以实 现多方向文字的检测. 高效准确自然文字检测器 (efficient and accurate scene text detector, EAST) ${ }^{[8]}$ 方法通过像素级回归获得了较好的检测结果，同 时也可以检测多方向的文字区域. 在检测过程中, 旋转敏感回归检测 (rotation-sensitive regression detector, RRD) ${ }^{[9]}$ 将旋转不变性特征用于分类, 将 旋转感知性特征用于回归，因此在检测长文字区 域时具有较好的性能. 但是, 这些方法都有共同的 局限性, 即由于边界框或顶点围成的四边形的形 状限制，它们在检测不规则形状文字区域(如曲文) 时表现不佳. 因为在自然场景中大量存在不规则 形状文字，所以有必要提出相关解决方法. 
基于分割的方法将像素级预测结果与后处理 算法相结合来检测文字区域. 为了获得准确的文 字区域检测结果，通常需要采用复杂的后处理过 程. Mask TextSpotter ${ }^{[10]}$ 采用基于掩膜区域卷积神 经网络 (mask region-based convolutional neural network, Mask RCNN)的实例分割方法检测任意形 状的文字实例. PSENet 通过以不同比例的内核对 图像中的文字区域进行收缩，避免了 2 个文字区域 由于距离太近而被错误地检测为一个更大文字区 域的情况. TextField ${ }^{[11]}$ 将文字方向作为特征之一在 网络中进行训练, 也可以避免将 2 个距离相近的文 字区域检测为一个文字区域的情况. 虽然这些方 法具有较高的准确度，同时适用于检测不规则形 状文字的工作，但复杂的后处理过程降低了其检 测速度.

\section{2 本文方法}

\section{1 概 述}

本文方法的总体流程如图 1 所示. 其中，黄色 箭头表示 2 倍下采样; 紫色箭头表示 2 倍上采样; 红 色箭头表示 4 倍上采样. 骨干网络由改进 ResNet ${ }^{[12]}$ 得到，在原始 ResNet 的 res-5 层，通过移除下采样 层保持了特征图的空间分辨率，同时使用扩张卷 积保持后续层的感受野，这种改进方式借鉴了扩 张残差网络(dilated residual network, DRN) ${ }^{[13]}$ 的思 路. 本文将改进后的骨干网络命名为 D5RN(dilated fifth-layer residual network), 其由低到高的 5 层分 别为 res-1, res-2, res-3, res-4 和 DRN-5. 通过改进, 在之后的注意力模块中，不会因进一步的卷积使 特征图分辨率降得太低导致丢失细节信息. 改进 的 FPA 级联在 DRN-5 层上, 3 个 GAU 分别将低层 特征信息和高层特征信息结合, FPA 和 GAU 的具

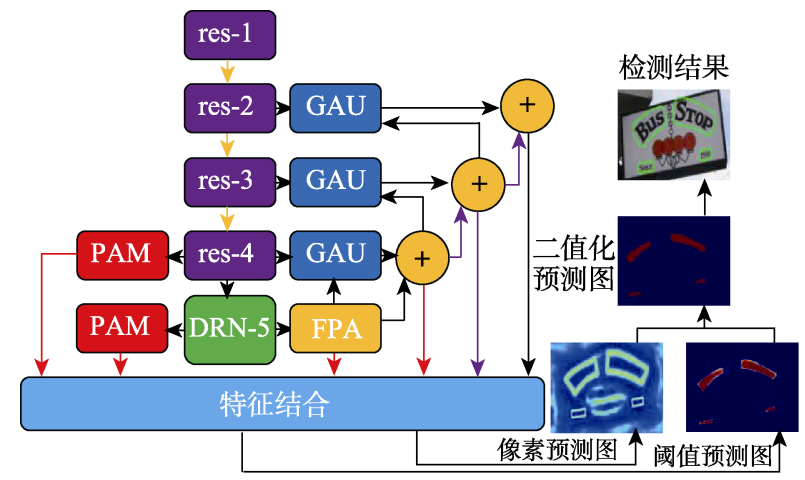

图 1 本文方法总体流程
体结构和作用将在下文展开介绍. 本文提出的应 用 PAN 特征提取网络和应用常用的 FPN 特征提取 网络各有其优势. PAN 由于引人了注意力机制, 实 现了更好的特征提取效果, 但其结构不像 FPN 那 样轻量化. 在实验过程中, 可根据骨干网络的深度 以及对检测准确度和检测速度的需求，灵活地选 择采用 PAN 还是 FPN 作为特征提取网络, 以达到 较好的综合性能.

\subsection{PAM}

PAM 采用自注意机制捕捉特征图中任意 2 个 位置之间的空间依赖关系, 其结构如图 2 所示. 对 于某一特定位置的特征，通过加权聚合图中所有 位置的特征更新该位置的特征, 各个位置与该位 置之间的特征相似性确定了对应的权重. PAM 使 得任何 2 个特征相似的位置, 无论它们在空间维度 上的距离如何, 都可以相互增强各自特征的表达.

如图 2 所示, PAM 输人为特征图 $\boldsymbol{A}(C \times H \times W)$, 其中, $C$ 为特征图通道数, $H$ 为特征图高度, $W$ 为特征图宽度. 用 3 个卷积层分别对 $\boldsymbol{A}$ 进行处理, 得到 3 个特征图 $\boldsymbol{X}, \boldsymbol{Y}$ 和 $\boldsymbol{Z}$. 然后将其尺寸重塑 为 $C \times N$, 其中 $N=H \times W$, 将重塑后的 $\boldsymbol{X}$ 进行转 置并与重塑后的 $\boldsymbol{Y}$ 相乘，得到的结果用 Softmax 进行处理后得到空间注意力图 $\boldsymbol{S}(N \times N)$. 这一部 分计算的意义是对各通道分别计算 $N$ 个像素两两 之间的相似性，然后将各通道信息结合得到 $\boldsymbol{S}$, 用来指导以其他点的像素对图中各点像素进行增 强. 具体实现为将 $\boldsymbol{S}$ 进行转置并与 $\mathbf{Z}$ 和比例系数 相乘, 将得到的结果重塑为原始尺寸, 最后与 $\boldsymbol{A}$

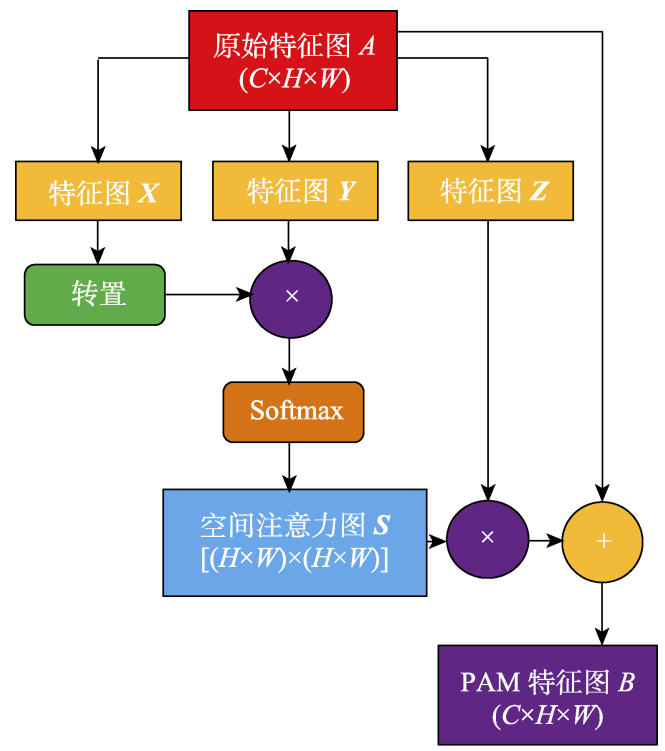

图 2 PAM 结构 
相加得到最终的输出 $\boldsymbol{B}(C \times H \times W)$. 可以看到, $\boldsymbol{B}$ 中每个像素的值都是所有像素原始特征的加权和.

\subsection{FPA 和 GAU}

本文采用的 PAN 由 FPA 和 GAU 组成. FPA 的 结构如图 3 所示.

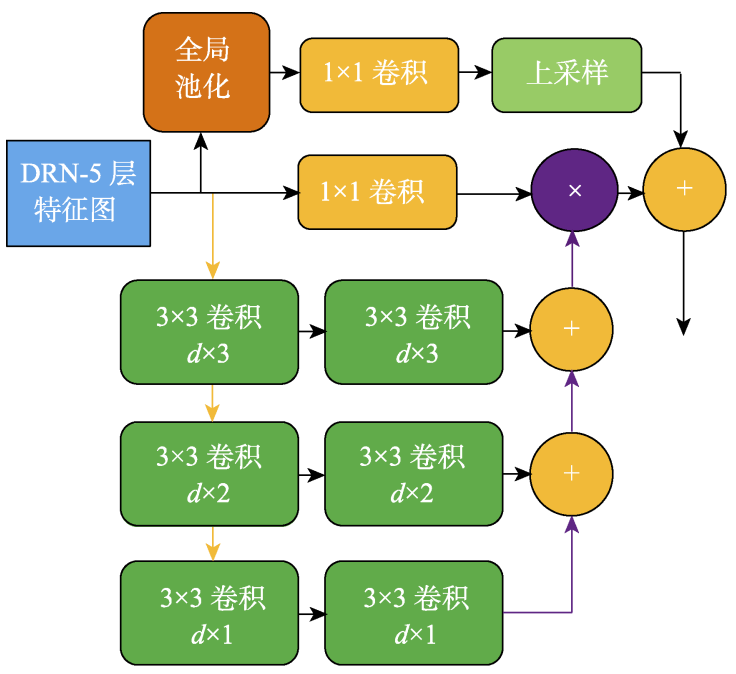

图 3 FPA 结构

图 3 中, 黄色箭头表示 2 倍下采样, 紫色箭头 表示 2 倍上采样, $d$ 表示空洞卷积的空洞系数. DRN-5 层得到的特征图分别进人 3 条支路进行处 理. 第 1 条支路是分别进行不同的卷积得到不同尺 寸的特征信息，然后将小尺寸的特征图依次进行 上采样并与大尺寸特征图结合. 其中, 使用不同步 长的空洞卷积是为了在增大感受野的同时减少计 算量. 第 2 条支路是对 DRN-5 层特征图进行 $1 \times 1$ 卷积. 第 3 条支路是对 DRN-5 层特征图依次进行 全局池化、 $1 \times 1$ 卷积、上采样. 将第 1 条支路得 到的特征与第 2 条支路得到的特征相乘，然后与第 3 条支路得到的特征结合, 得到 FPA 的输出. FPA 为顶层特征图的每一个通道都产生了一个空间注 意力图, 且其融合了针对不同尺度的 3 条支路产生 的注意力图，同时，通过加人全局信息，减轻了类 内异质性. FPA 的作用是提供像素级注意力信息 并通过金字塔结构扩大感受野的范围。

在本文的网络中, 3 个 $\mathrm{GAU}$ 依次将低层信息与 高层信息结合, GAU 的结构如图 4 所示.

图 4 中, 紫色箭头表示 2 倍上采样. 对于每个 高层特征图, 分别进行 2 条支路的处理, 第 1 条支 路将高层特征图分别进行全局池化和 $1 \times 1$ 卷积, 并与它下一层的低层特征图经过 $3 \times 3$ 卷积后得到 的特征相乘，第 2 条支路将高层特征图进行 2 倍上

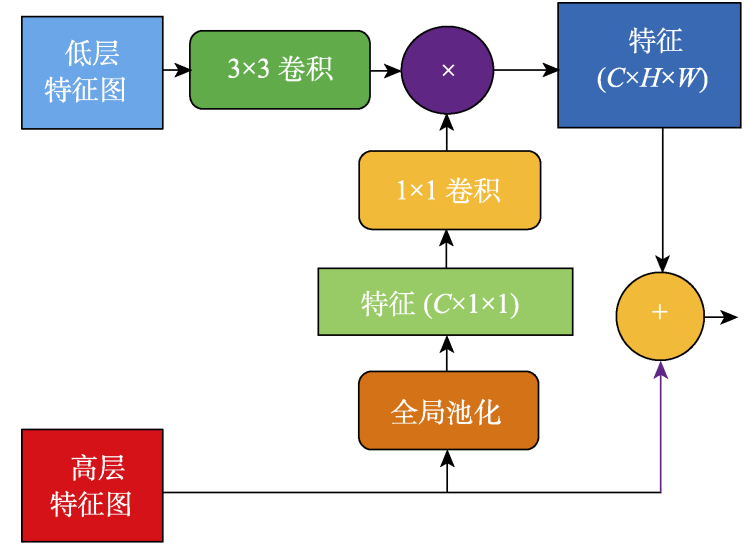

图 $4 \mathrm{GAU}$ 结构

采样, 最后将 2 条支路得到的结果结合. GAU 的作 用是利用高层特征产生对低层特征的通道指导注 意力信息，指导低层特征恢复图像像素的定位.

\section{4 自适应二值化}

最终得到的特征图进人预测网络得到 2 种图. 一种为像素预测图, 用来表示每个像素上文本/非 文本的概率; 另一种为阈值预测图，用来表示每个 像素上的二值化阈值, 在训练时可对其进行优化. 最后将像素预测图和阈值预测图结合, 通过自适 应二值化得到二值化预测图.

在传统的基于分割的文字检测网络中，最终 的二值化预测图是通过固定阈值获得的, 选取 的阈值不同对检测结果影响较大. 二值化预测 公式为

$$
B_{m, n}=\left\{\begin{array}{ll}
1, & \text { if } P_{m, n} \geqslant t \\
0, & \text { otherwise }
\end{array} .\right.
$$

其中, $B_{m, n}$ 和 $P_{m, n}$ 分别表示坐标为 $(m, n)$ 的像素 的二值化预测值和像素预测值; $t$ 为固定的二值 化阈值.

而本文采用的自适应二值化公式为

$$
\left\{\begin{array}{l}
\tilde{B}_{m, n}=\tanh \left[k\left(P_{m, n}-T_{m, n}\right)\right] \\
B_{m, n}=0.5 \times \tilde{B}_{m, n}+0.5
\end{array}\right.
$$

其中, $\tilde{B}_{m, n}$ 和 $B_{m, n}$ 分别表示归一化前后的二值化 预测值; $P_{m, n}$ 和 $T_{m, n}$ 分别表示坐标为 $(m, n)$ 的像素 的像素预测值和阈值预测值.

用式(1)代替固定阈值二值化公式，将二值化 操作变为可微操作, 可以将每一个像素点进行自 适应二值化, 且通过网络学习得到二值化阈值. 将 二值化这一后处理过程加人网络一起训练, 极大 地增强了最终输出结果图对于阈值的鲁棒性.

\section{5 标签制作}

在标签制作过程中，本文采用 Vatti 裁剪算法, 
其裁剪偏移量计算公式为 $D=\frac{A\left(1-r^{2}\right)}{L}$. 其中, $L$ 为周长; $A$ 为面积; $r$ 为收缩比例.

将训练集图像中文字区域的 Ground Truth 经 过 $r=0.7$ 的 Vatti 裁剪得到区域 $G_{\mathrm{c}}$, 将其作为小尺 寸中心区域像素标签，同时将 Ground Truth 以同样 的裁剪偏移量 $D$ 扩张为 $G_{\mathrm{d}}$, 由 $G_{\mathrm{d}}$ 和 $G_{\mathrm{c}}$ 中间的区 域可以得到小尺寸边界区域阈值标签.

\section{6 由二值化预测图得到文字区域}

检测过程的最后一步是由二值化预测图得到 文字区域检测结果. 由于本文采用以 0.7 的比例进 行裁剪得到的文字中心区域标签进行训练, 因此 在检测中需要以对应得到的文字中心区域二值化 预测结果为膨胀内核, 采用 Vatii 裁剪算法进行膨 胀操作以得到标准文字区域的检测结果. 膨胀偏 移量的计算公式为 $D^{\prime}=\frac{A^{\prime} \times r^{\prime}}{L^{\prime}}$. 其中, $A^{\prime}$ 为内核 的面积; $L^{\prime}$ 为内核的周长; $r^{\prime}$ 为膨胀比例, 其根 据先验知识预先设置.

\section{3 实验与结果}

\section{1 数据集}

本文实验所采用的数据集包括 SynthText ${ }^{[14]}$, Total-text ${ }^{[15]}$ 和 MSRA-TD500 ${ }^{[16]}$.

SynthText 是一个由 8000 幅背景图像合成的、 包含 800 万幅图像的数据集, 本文应用其预训练 模型.

Total-text 数据集包含 1255 幅训练图像和 300 幅测试图像. 其中包含了各种形状的文本，包括水 平文本、多方向文本和弯曲文本. 因此, 该数据集 适用于测试各方法对不规则形状文字的检测性能.

MSRA-TD500 数据集包含 300 幅训练图像和 200 幅测试图像, 是一个包含英文和中文的多语言 数据集. 其图像具有多样性, 能够很好地测试各方 法的性能.

\section{2 损失函数}

损失函数 $L_{\text {total }}$ 可以表示为像素预测图的损失 $L_{\mathrm{p}}$ 、阈值预测图的损失 $L_{\mathrm{t}}$ 和二值化预测图的损失 $L_{\mathrm{b}}$ 的加权和, 即 $L_{\text {total }}=L_{\mathrm{p}}+\alpha \times L_{\mathrm{b}}+\beta \times L_{\mathrm{t}}$. 根据先 验知识及实验测试, 将 $\alpha$ 和 $\beta$ 分别设置为 1 和 10 .

$L_{\mathrm{p}}$ 和 $L_{\mathrm{b}}$ 均采用二值化交叉熵损失函数, 为了 解决正负样本数目不平衡的问题, 在训练中采用 难例挖掘策略. $L_{\mathrm{p}}$ 和 $L_{\mathrm{b}}$ 的公式为

$$
L_{\mathrm{p}}=L_{\mathrm{b}}=\sum_{i \in S_{1}} y_{i} \ln x_{i}+\left(1-y_{i}\right) \ln \left(1-x_{i}\right) .
$$

其中, $S_{1}$ 为经过采样的样本集, 正负样本的比例 为 $1: 3$.

$L_{\mathrm{t}}$ 由计算预测结果和标签中各对应像素点的 $L_{1}$ 范数的和得到, 即 $L_{\mathrm{t}}=\sum_{i \in R_{\mathrm{d}}}\left|y_{i}^{*}-x_{i}^{*}\right|$. 其中, $R_{\mathrm{d}}$ 为 标签中扩张区域 $G_{\mathrm{d}}$ 中像素点的集合.

\section{3 实验细节}

为了提高网络性能, 本文在骨干网络中采用 了可变形卷积 ${ }^{[17]}$. 可变形卷积为该模型提供了一 个灵活的感受野，尤其在长宽比非常大的文字区 域的检测中效果更加显著.

实验过程中, 首先使用 SynthText 数据集对模 型进行了 10 万次迭代的预训练, 然后在各个实验 数据集上进行了 1000 个 epoch 的训练, 以优化模 型. 在实验中采用了几种数据增加的方法, 包括 角度范围为 $\left(-10^{\circ}, 10^{\circ}\right)$ 的随机旋转、随机剪切和随 机翻转.

\section{4 在 Total-text 数据集上的实验结果}

首先采用由 ResNet-18 改进得到的 D5RN-18 作为骨干网络进行实验，同时采用 PAN 作为特征 提取网络, 并采用 PAM. 在测试阶段, 将图像的宽 度设置为 896 像素, 并利用固定宽高比对高度进行 调整.

将本文方法与其他方法在 Total-text 数据集上 进行比较, 结果如表 1 所示.

表 1 不同方法在 Total-text 数据集上的实验结果

\begin{tabular}{|c|c|c|c|c|}
\hline 方法 & 精确率/\% & 召回率/\% & $F$ 值 $/ \%$ & 速度 $/\left(\right.$ 帧 $\left.\cdot \mathrm{s}^{-1}\right)$ \\
\hline TextSnake $^{[18]}$ & 82.7 & 74.5 & 78.4 & \\
\hline 文献[19] & 80.9 & 76.2 & 78.5 & \\
\hline TextSpotter ${ }^{[10]}$ & 82.5 & 75.6 & 78.6 & \\
\hline TextField $^{[11]}$ & 81.2 & 79.9 & 80.6 & \\
\hline 文献[20] & 87.6 & 79.3 & 83.3 & \\
\hline CRAFT $^{[21]}$ & 87.6 & 79.9 & 83.6 & \\
\hline 文献[22] & 81.4 & 79.1 & 80.2 & \\
\hline PSENet $^{[1]}$ & 84.0 & 78.0 & 80.9 & 3.9 \\
\hline 本文 D5RN-18 & 86.7 & 79.6 & 83.0 & 27.0 \\
\hline 本文 D5RN-50 & 88.1 & 83.3 & 85.6 & 24.0 \\
\hline
\end{tabular}

注. 粗体表示各项指标里的最优结果.

首先，本文以 D5RN-18 作为骨干网络在检测 速度和检测准确度上都取得了很好的性能. 与 PSENet 相比, 本文方法的检测速度大幅领先于 PSENet, 而 $F$ 值为 $83.0 \%$, 比 PSENet 高 $2.1 \%$. 与 
其他方法相比, 本文方法的 $F$ 值仍有较强竞争力, 同时得益于 D5RN-18 骨干网络的轻量化和更高效 的后处理算法，本文方法在检测速度上有很大的 优势. 因此, 本文方法显示出其良好的综合性能.

然后采用由 ResNet-50 改进得到的 D5RN-50 作为骨干网络进行实验，同样采用 PAM，考虑实 现较高的检测速度和准确度的综合性能，不采用 PAN 作为特征提取网络, 而是采用 FPN 代替, 且 图像分辨率的设置原理与上一个实验相同.

实验结果如表 1 所示, 与其他方法相比, 本文 方法在准确度和速度上都有很好的表现. 该方法 实现了 $85.6 \%$ 的 $F$ 值，准确度比字符区域感知文字 检测(character region awareness for text-detection, CRAFT $)^{[21]}$ 方法提高了 $2 \%$, 在进行对比的各种方 法中较为领先, 而且检测速度与其他方法相比也 有很大优势.

\section{5 在 MSRA-TD500 数据集上的实验结果}

本文同样在 MSRA-TD500 数据集上进行了实 验，以更全面地测试所提方法的性能. 网络结构与 之前的实验相同，在实验过程中, 将 HUST-TR400 $0^{[23]}$ 数据集中的 400 幅训练图像加人训练集中一起训练, 训练图像的分辨率已被设置为 $640 \times 640$. 在测试中, 将测试图像宽度调整为 768 像素，高度按固定宽高 比进行调整. 将本文方法与其他方法进行对比，结 果如表 2 所示.

表 2 不同方法在 MSRA-TD50 上的实验结果对比

\begin{tabular}{|c|c|c|c|c|}
\hline 方法 & 精确率/\% & 召回率/\% & $F$ 值 $/ \%$ & 速度/(帧· $\left.\mathrm{s}^{-1}\right)$ \\
\hline 文献[24] & 77.0 & 70.0 & 74.0 & 1.1 \\
\hline 文献[25] & 82.0 & 68.0 & 74.0 & \\
\hline $\mathrm{RRD}^{[9]}$ & 80.2 & 73.9 & 76.9 & 7.7 \\
\hline PixelLink ${ }^{[26]}$ & 83.0 & 73.2 & 77.8 & 3.0 \\
\hline 文献[27] & 87.6 & 76.2 & 81.5 & 5.7 \\
\hline 文献[28] & 88.0 & 79.0 & 83.0 & \\
\hline TextSnake ${ }^{[18]}$ & 83.2 & 73.9 & 78.3 & 1.1 \\
\hline CRAFT $^{[21]}$ & 88.2 & 78.2 & 82.9 & 8.6 \\
\hline 文献[29] & 84.2 & 81.7 & 82.9 & \\
\hline 本文 D5RN-18 & 89.1 & 78.0 & 83.2 & 34.0 \\
\hline 本文 D5RN-50 & 91.4 & 79.6 & 85.1 & 23.0 \\
\hline
\end{tabular}

注. 粗体表示各项指标里的最优结果.

如表 2 所示，本文方法分别使用 D5RN-18 和 D5RN-50 作为骨干主干网络时在速度和准确度方 面都有较大优势. 与其他方法相比，本文方法使用 D5RN-18 作为骨干网络时实现了较高的检测准确 度; 当使用 D5RN-50 作为骨干网络时, 其 $F$ 值比
CRAFT 高 2.2\%, 且本文方法在检测速度上对比其 他方法有着显著的、倍数级的优势. 因此, 本文方 法在检测速度和准确度的综合性能上取得了领先.

\section{6 关于 PAM 的消融实验}

本文在 Total-text 数据集上进行了消融实验, 以验证 PAM 的作用. 在作为对比的 2 组实验中, 每组均分别采用 D5RN-18 和 D5RN-50 骨干网络. 因为只验证 PAM 对检测准确度的提升, 所以不采 用 PAN, 每组实验均以 FPN 作为特征提取网络, 实验结果如表 3 所示.

表 3 不同骨干网络是否采用 PAM 的实验结果对比

\begin{tabular}{|c|c|c|c|c|c|}
\hline 骨干网络 & PAM & 精确率/\% & 召回率/\% & $F$ 值 $/ \%$ & 速度/(帧 $\left.\cdot \mathrm{s}^{-1}\right)$ \\
\hline D5RN-18 & $\sqrt{ }$ & 86.5 & 79.2 & 82.7 & 40.0 \\
\hline D5RN-18 & $x$ & 86.9 & 77.8 & 82.1 & 46.0 \\
\hline D5RN-50 & $\sqrt{ }$ & 88.1 & 83.3 & 85.6 & 24.0 \\
\hline D5RN-50 & $x$ & 86.6 & 81.6 & 84.0 & 31.0 \\
\hline
\end{tabular}

从表 3 中可以看出, 在使用 D5RN-18 骨干网络 时, PAM 可以使 $F$ 值提高 $0.6 \%$; 在使用 D5RN-50 骨干网络时, PAM 可以使 $F$ 值提高 $1.6 \%$, 因此可 以得出以下结论：PAM 对检测准确度的提升是显 著的，且对检测速度的影响并不大.

\subsection{PAN 和 FPN 的对比实验}

本文在 Total-text 数据集上进行了对比实验, 验证 PAN 的作用以及对比 2 种特征提取网络的优 势. 在作为对比的 2 组实验中, 分别采用 PAN 和 FPN 作为特征提取网络, 每组实验分别采用 D5RN-18 和 D5RN-50 骨干网络, 同时采用 PAM. 实验结果如表 4 所示.

\begin{tabular}{cccccc} 
表 4 & \multicolumn{6}{c}{ 不同骨干网络采用 PAN 和 FPN 的实验结果 } \\
\hline \multirow{2}{*}{ 骨干网络 } & $\begin{array}{c}\text { 特征提 } \\
\text { 取网络 }\end{array}$ & $\begin{array}{c}\text { 精确 } \\
\text { 率/\% }\end{array}$ & $\begin{array}{c}\text { 召回 } \\
\text { 率/\% }\end{array}$ & $\begin{array}{c}F \text { 值/ } \\
\%\end{array}$ & $\begin{array}{c}\text { 速度/ } \\
\left(\text { (帧· } \mathrm{s}^{-1} \text { ) }\right.\end{array}$ \\
\hline D5RN-18 & FPN & 86.5 & 79.2 & 82.7 & 40.0 \\
D5RN-18 & PAN & 86.7 & 79.6 & 83.0 & 27.0 \\
D5RN-50 & FPN & 88.1 & 83.3 & 85.6 & 24.0 \\
D5RN-50 & PAN & 88.2 & 83.3 & 85.7 & 15.0 \\
\hline
\end{tabular}

从表 4 中可以看出, 以 $\mathrm{D} 5 \mathrm{RN}-18$ 作为骨干网 络时, 采用 FPN 可以使 $F$ 值提高 $0.3 \%$, 同时依旧 可以实现较高的检测速度, 因此选择 PAN 作为特 征提取网络是更好的选择. 以 D5RN-50 作为骨干 网络时, 采用 PAN 同样可以提高 $F$ 值, 从而实现 $F$ 值的进一步领先, 然而与此同时降低了检测速 度. 由于骨干网络层数的加深会导致检测速度的 降低, 出于对检测准确度和检测速度 2 项指标的综 
合性能的考量, 本文在以 D5RN-50 作为骨干网络 时选择采用 FPN 作为特征提取网络.

\section{4 结 语}

在自然场景文字检测中，检测准确度和检测 速度是 2 项重要的指标. 检测准确度保证了检测结 果的可靠性，而检测速度则决定了检测的效率，而 目前的检测方法中大多难以同时实现较高的检测 准确度和较快的检测速度. 本文提出将注意力机 制应用于自然场景文字检测中，采用 PAM 和 PAN 提升检测准确度，同时使用较为简单的后处理过 程，使本文方法在这 2 个指标上都有着较好的表 现. 在各个数据集上的实验显示, 本文方法可以在 $F$ 值和检测速度 2 项指标中的其中一项指标达到 领先的同时，另一项指标仍保持较高，即实现了检 测准确度和检测速度的综合性能上的优势。

\section{参考文献(References):}

[1] Wang W H, Xie E Z, Li X, et al. Shape robust text detection with progressive scale expansion network[C] //Proceedings of the IEEE/CVF Conference on Computer Vision and Pattern Recognition. Los Alamitos: IEEE Computer Society Press, 2019: 9336-9345

[2] Vatti B R. A generic solution to polygon clipping[J]. Communications of the ACM, 1992, 35(7): 56-64

[3] Fu J, Liu J, Tian H J, et al. Dual attention network for scene segmentation[C] //Proceedings of the IEEE/CVF Conference on Computer Vision and Pattern Recognition. Los Alamitos: IEEE Computer Society Press, 2019: 3146-3154

[4] Li H C, Xiong P F, An J, et al. Pyramid attention network for semantic segmentation[OL]. [2020-10-08]. https://arxiv.org/ abs/1805.10180v3

[5] Liao M H, Wan Z Y, Yao C, et al. Real-time scene text detection with differentiable binarization[C] //Proceedings of the AAAI Conference on Artificial Intelligence. Palo Alto: AAAI Press, 2020: 11474-11481

[6] Liu W, Anguelov D, Erhan D, et al. SSD: single shot multibox detector[C] //Proceedings of European Conference on Computer Vision. Heidelberg: Springer, 2016: 21-37

[7] Liao M H, Shi B G, Bai X. TextBoxes++: a single-shot oriented scene text detector[J]. IEEE Transactions on Image Processing, 2018, 27(8): 3676-3690

[8] Zhou X Y, Yao C, Wen H, et al. EAST: an efficient and accurate scene text detector[C] //Proceedings of the IEEE Conference on Computer Vision and Pattern Recognition. Los Alamitos: IEEE Computer Society Press, 2017: 5551-5560

[9] Liao M H, Zhu Z, Shi B G, et al. Rotation-sensitive regression for oriented scene text detection[C] //Proceedings of the
IEEE/CVF Conference on Computer Vision and Pattern Recognition. Los Alamitos: IEEE Computer Society Press, 2018: 5909-5918

[10] Lyu P Y, Liao M H, Yao C, et al. Mask TextSpotter: an end-to-end trainable neural network for spotting text with arbitrary shapes[C] //Proceedings of European Conference on Computer Vision. Heidelberg: Springer, 2018: 71-88

[11] Xu Y C, Wang Y K, Zhou W, et al. TextField: learning a deep direction field for irregular scene text detection[J]. IEEE Transactions on Image Processing, 2019, 28(11): 5566-5579

[12] He K M, Zhang X Y, Ren S Q, et al. Deep residual learning for image recognition[C] //Proceedings of the IEEE Conference on Computer Vision and Pattern Recognition. Los Alamitos: IEEE Computer Society Press, 2016: 770-778

[13] Yu F, Koltun V, Funkhouser T. Dilated residual networks[C] //Proceedings of the IEEE Conference on Computer Vision and Pattern Recognition. Los Alamitos: IEEE Computer Society Press, 2017: 472-480

[14] Gupta A, Vedaldi A, Zisserman A. Synthetic data for text localisation in natural images $[\mathrm{C}] / /$ Proceedings of the IEEE Conference on Computer Vision and Pattern Recognition. Los Alamitos: IEEE Computer Society Press, 2016: 2315-2324

[15] Chng C K, Chan C S. Total-text: a comprehensive dataset for scene text detection and recognition[C] //Proceedings of the 14th IAPR International Conference on Document Analysis and Recognition. New York: IAPR Press, 2017: 935-942

[16] Yao C, Bai X, Liu W Y, et al. Detecting texts of arbitrary orientations in natural images[C] //Proceedings of the IEEE Conference on Computer Vision and Pattern Recognition. Los Alamitos: IEEE Computer Society Press, 2012: 1083-1090

[17] Dai J F, Qi H Z, Xiong Y W, et al. Deformable convolutional networks[C] //Proceedings of the IEEE International Conference on Computer Vision. Los Alamitos: IEEE Computer Society Press, 2017: 764-773

[18] Long S B, Ruan J Q, Zhang W J, et al. TextSnake: a flexible representation for detecting text of arbitrary shapes[C] // Proceedings of European Conference on Computer Vision. Heidelberg: Springer, 2018: 19-35

[19] Wang X B, Jiang Y Y, Luo Z B, et al. Arbitrary shape scene text detection with adaptive text region representation[C] //Proceedings of the IEEE/CVF Conference on Computer Vision and Pattern Recognition. Los Alamitos: IEEE Computer Society Press, 2019: 6449-6458

[20] Zhang C Q, Liang B R, Huang Z M, et al. Look more than once: an accurate detector for text of arbitrary shapes[C] //Proceedings of the IEEE/CVF Conference on Computer Vision and Pattern Recognition. Los Alamitos: IEEE Computer Society Press, 2019: 10552-10561

[21] Baek Y, Lee B, Han D, et al. Character region awareness for text detection[C] //Proceedings of the IEEE/CVF Conference on Computer Vision and Pattern Recognition. Los Alamitos: IEEE Computer Society Press, 2019: 9365-9374

[22] Liu Y L, Jin L W, Zhang S T, et al. Curved scene text detection via transverse and longitudinal sequence connection[J]. Pattern Recognition, 2019, 90: 337-345 
[23] Yao C, Bai X, Liu W Y. A unified framework for multioriented text detection and recognition[J]. IEEE Transactions on Image Processing, 2014, 23(11): 4737-4749

[24] He W H, Zhang X Y, Yin F, et al. Deep direct regression for multi-oriented scene text detection[C] //Proceedings of the IEEE International Conference on Computer Vision. Los Alamitos: IEEE Computer Society Press, 2017: 745-753

[25] Ma J Q, Shao W Y, Ye H, et al. Arbitrary-oriented scene text detection via rotation proposals[J]. IEEE Transactions on Multimedia, 2018, 20(11): 3111-3122

[26] Deng D, Liu H F, Li X L, et al. PixelLink: detecting scene text via instance segmentation[C] //Proceedings of the AAAI Conference on Artificial Intelligence. Palo Alto: AAAI Press, 2018: 6773-6780
[27] Lyu P Y, Yao C, Wu W H, et al. Multi-oriented scene text detection via corner localization and region segmentation[C] //Proceedings of the IEEE/CVF Conference on Computer Vision and Pattern Recognition. Los Alamitos: IEEE Computer Society Press, 2018: 7553-7563

[28] Liu Z C, Lin G S, Yang S, et al. Learning Markov clustering networks for scene text detection[C] //Proceedings of the IEEE/CVF Conference on Computer Vision and Pattern Recognition. Los Alamitos: IEEE Computer Society Press, 2018: 6936-6944

[29] Tian Z T, Shu M, Lyu P Y, et al. Learning shape-aware embedding for scene text detection[C] //Proceedings of the IEEE/CVF Conference on Computer Vision and Pattern Recognition. Los Alamitos: IEEE Computer Society Press, 2019: 4234-4243 